\title{
Influencia del clima, uso del suelo y profundidad sobre el contenido de carbono orgánico en dos pisos altitudinales andinos del departamento Norte de Santander, Colombia
}

\section{Influence of climate, soil use and soil depth on soil organic carbon content at two Andean altitudinal sites in Norte de Santander, Colombia}
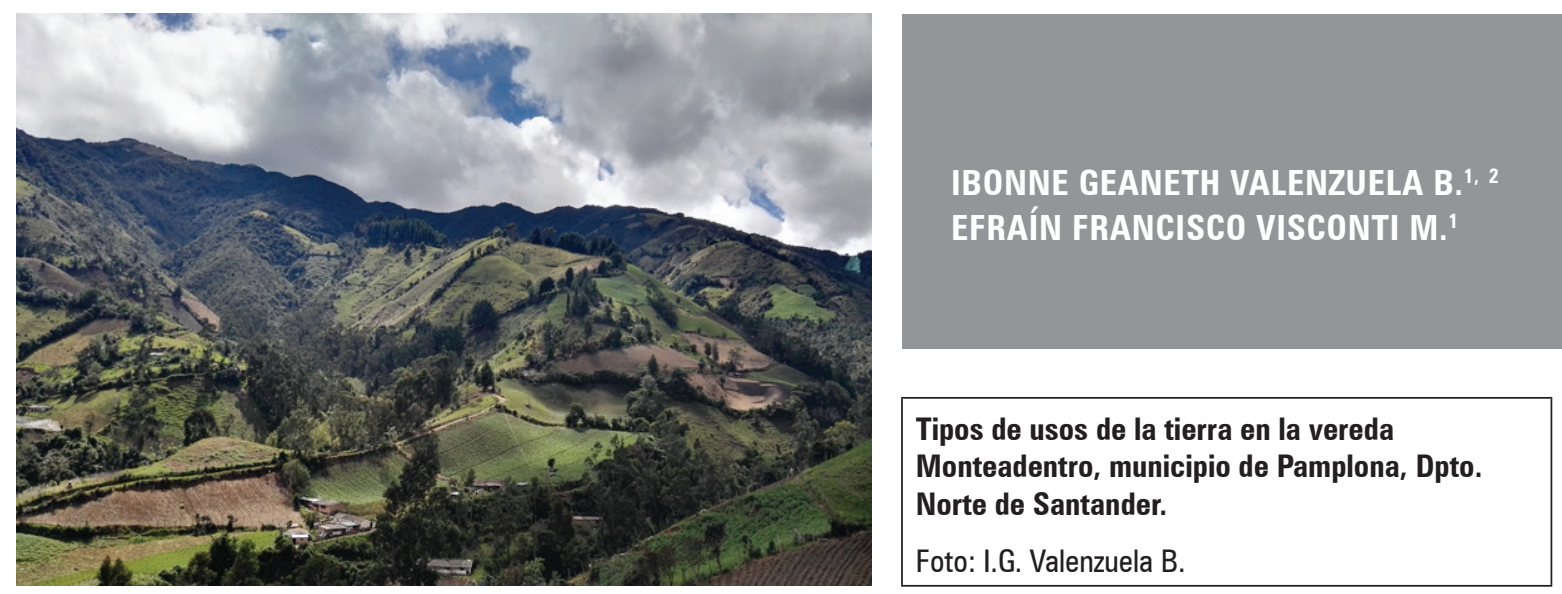

\section{RESUMEN}

El incremento y estabilización del carbono orgánico del suelo (COS) representa una alternativa viable para la mitigación del efecto invernadero. Pero el COS es severamente afectado por los cambios del suelo, existiendo controversias sobre cuáles tipos de uso de la tierra favorecen el secuestro de carbono. El clima tiene efecto importante sobre la dinámica del COS, por tanto el efecto generado por el uso y manejo del suelo es distinto en climas fríos y cálidos. Para entender como los factores clima, uso del suelo y profundidad del suelo, afectan la dinámica del COS en dos lugares del departamento de Norte de Santander en Colombia, se evaluaron propiedades físicas (arenas, limos y arcillas, densidad aparente) y propiedades químicas ( $\mathrm{pH}$, conductividad eléctrica, capacidad de intercambio catiónico y saturación de bases). Se determinó el contenido de carbono orgánico total (COT), fracción ligera (CO de FL), fracción húmica (CO de FH) y biomasa microbiana (CO de $\mathrm{BM}$ ). Estas evaluaciones se realizaron en dos climas contrastantes (frío y cálido), tres tipos de uso del suelo (bosque, pasturas y cultivo intensivo) y tres profundidades ( 0 a $5 \mathrm{~cm}, 5$ a $10 \mathrm{~cm}$ y 10 a $20 \mathrm{~cm}$ ). Se encontró que hay mayor carbono orgánico en todos los compartimientos (COT, CO de BM, CO de FL y CO de FH) del suelo en clima frío. Los usos del suelo en bosque y pastura, son más favorables para el almacenamiento de COS en clima frío, mientras en cultivo intensivo genera menor contenido de COT, CO de FL y CO de BM. El índice de humificación y el CO de FH fueron mayores en cultivo intensivo de clima cálido.

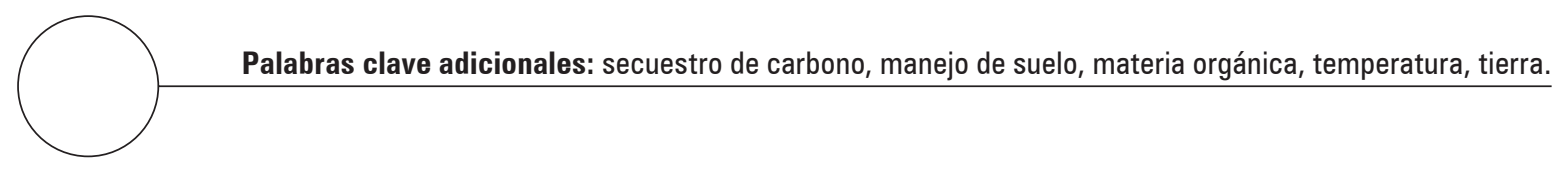

Facultad de Ciencias Agrarias y del Ambiente, Departamento de Ciencias del Medio Ambiente, Grupo de investigación Ambiente y Vida, Universidad Francisco de Paula Santander, Cúcuta (Colombia). ORCID Valenzuela B., I.G.: 0000-0002-1173-3133; ORCID Visconti M., E.F.: 0000-0002-7017-313X

2 Autor para correspondencia. ibonnegeanethvb@ufps.edu.co 


\section{ABSTRACT}

The increase and stabilization of soil organic carbon (SOC) represent a feasible alternative for mitigating greenhouse effect. But, SOC is severely affected by changes in soil use, and there is uncertainty about which types of soil use favor carbon sequestration. In addition, climate influences SOC dynamics; therefore, the effect generated by the use and management of soils is different in cold climates and warm climates. In order to understand how climate, soil use and soil depth influence SOC dynamics at two Andean sites in Norte de Santander, Colombia, the soil physical (sands, silts, clays, and bulk density) and chemical (pH, E.C., C.E.C. and S.B) properties were studied. Also, the total organic carbon (TOC), light fraction (LF), humic fraction (HF), microbiol biomass (MB) and organic carbon were measured. These evaluations were performed in two climates (cold and warm), with three soil uses per climate and three soil depths ( 0 to $5 \mathrm{~cm}, 5$ to $10 \mathrm{~cm}$ and 10 to $20 \mathrm{~cm}$ ). The amount of organic carbon was statistically higher in the cold climate soils for all compartments (TOC, OC of MB, OC of LF and OC of HF). The Forest and Pasture were more favorable for SOC storage in the cold climates, and the intensive crops presented a lower content of TOC, OC of $\mathrm{MB}$ and $\mathrm{OC}$ of LF. The humification rate and OC of HF were higher in the soils with intensive crops as the soil use in the warm climate.

Additional keywords: carbon sequestration, soil management, organic matter, temperature, land.

Fecha de recepción: 07-08-2017 Aprobado para publicación: 30-01-2018

INTRODUCCIÓN

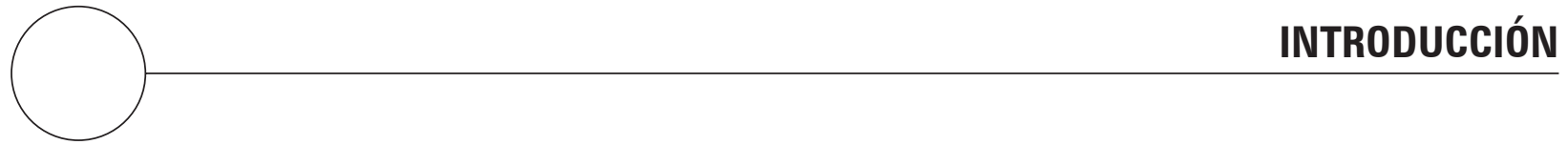

El Plan de Desarrollo para el departamento de Norte de Santander, 2016-2019, plantea un marco estratégico que establece la productividad como uno de los pilares del plan y propone dentro de las apuestas productivas la producción agroindustrial, específicamente en los subsectores (cultivos): cacao, palma de aceite, café, arroz, hortifrutícola y ganadería (Gobernación de Norte de Santander, 2016).

Por otra parte, los suelos representan el tercer compartimiento de nuestro planeta en almacenar carbono (C). Desde luego, ningún compartimiento es estático, pero es en la atmosfera donde el incremento de $\mathrm{C}$ representa riesgos por su efecto invernadero, mientras que en los suelos su presencia en las formas de carbono orgánico (CO), significa enormes beneficios por el incremento de la productividad de los mismos (Macías y Camps-Arbestain, 2010).

En este sentido, se señala que la materia orgánica del suelo (MOS) es la principal responsable de la capacidad que tienen los suelos en prestar servicios agrícolas y ambientales (Manlay et al., 2007).

Actualmente se entiende que la MOS es un compartimiento muy importante de los ecosistemas terrestres.
Desde el punto de vista químico y ecológico, la MOS se considera formada por un conjunto de fracciones donde el $\mathrm{CO}$ se encuentra en distintas proporciones. Para estudiar cada una de estas fracciones son necesarias distintas técnicas químicas y físicas para la separación, análisis e interpretación (Elliott y Cambardella, 1991).

El equilibrio en el contenido de carbono orgánico en cada fracción de la materia orgánica, se encuentra fuertemente influenciado por el clima, el material parental, la vegetación y el manejo del suelo (Manlay et al., 2007).

Los suelos cuentan con capacidad para acumular C, pero también pueden emitirlo a la atmosfera, por tanto, los suelos contribuyen a la regulación del ciclo de C y sus implicaciones en el cambio climático (Olson et al., 2014). El factor principal que genera la emisión de carbono en el suelo o acumulación en el mismo es la modificación de la cobertura natural del suelo. La mecanización de tierras ha causado una pérdida de $\mathrm{CO}$ y un aumento en las emisiones de C, al tiempo que la reforestación de tierras cultivadas reporta un aumento en el secuestro de C. El proceso de captura de $\mathrm{C}$ en el suelo debido a recuperación de bosques u 
otros usos no agrícolas es lento, en comparación con el CO perdido por el suelo en cultivos. Las diferencias de escala temporal entre pérdidas antrópicas y recuperación son generalmente de varios órdenes de magnitud, siendo las propiedades físicas de los suelos y el contenido de materia orgánica significativas al cambio climático (Ussiri y Lal, 2013).

El suelo se considera como uno de los recursos naturales con mayor potencial para mitigar el efecto invernadero al acumular $\mathrm{CO}$ pero es necesario comprender los procesos de intercambio de gases de efecto invernadero (GEI) entre el suelo y la atmosfera, así como los procesos de almacenamiento de carbono en el suelo, de ello depende en buena medida las opciones que pueden implementarse en la mitigación del efecto invernadero (Ghimire et al., 2017).

Algunas investigaciones han señalado que los sistemas agroforestales tienen la capacidad de mantener las condiciones de fertilidad del suelo y almacenar mayor cantidad de COS en comparación con los bosques naturales, a la vez que sirven de sumidero de carbono (Kassa et al., 2017); otros han señalado que las coberturas de vegetación natural almacenan mayor cantidad de COS (Ferreira et al., 2016). Ante estos resultados ambivalentes, es necesario que se adelanten los estudios que permitan conocer el comportamiento del COS en cada escenario.

En este sentido, se planteó estudiar en el departamento de Norte de Santander como se ha afectado el contenido de COS, debido al cambio de uso de la tierra de bosque natural a agro ecosistema en dos pisos térmicos altitudinales; que según la clasificación climática Caldas - Lang son: frío y cálido.

\section{MATERIALES Y MÉTODOS}

Los suelos estudiados se ubican en dos municipios del departamento de Norte de Santander (Colombia). Uno en la zona de alta montaña (clima frío), ubicado en la vereda Monteadentro del municipio de Pamplona; se escogieron tres tipos de uso de la tierra (TUT) en lotes colindantes (bosque, pasturas y cultivos hortícolas intensivos). Según IGAC (2006a), se encuentra dentro de la zona de vida de Bosque húmedo Premontano, temperatura media anual de $13,5^{\circ} \mathrm{C}$, precipitación promedio anual de $900 \mathrm{~mm}$, altitud aproximada de 2.558 msnm y ubicación: $7^{\circ} 20^{\prime} 47,59$ " N y $72^{\circ} 39^{\prime} 50,62^{\prime \prime}$ W.
El segundo lugar se localiza en la zona baja de un valle aluvial de clima cálido, ubicado en la vereda Astilleros del municipio El Zulia, donde se escogieron los TUT en lotes colindantes (bosque, palma de aceite con pastura y cultivo de arroz tecnificado). Según IGAC (2006a), se encuentra dentro de la zona de vida de Bosque húmedo Tropical, temperatura promedio de $27^{\circ} \mathrm{C}$, precipitación promedio anual $2.200 \mathrm{~mm}$, altitud aproximada 76 msnm y localizado: 8०12'13,5" N y $72^{\circ} 32^{\prime} 52,1^{\prime \prime} \mathrm{W}$.

Se efectuó el muestreo de suelo en cada uno de los lotes seleccionados, recolectando muestras disturbadas y no disturbadas. Se tomaron muestras de suelo a tres profundidades: 0 a $5 \mathrm{~cm}, 5$ a $10 \mathrm{~cm}$ y 10 a $20 \mathrm{~cm}$. Considerando que en términos generales conforma la capa arable o profundidad de laboreo del suelo (0 a $20 \mathrm{~cm}$ ). A la capa arable se le separa en tres capas, ya que en los primeros $5 \mathrm{~cm}$ se considera es donde se acumularía la mayor parte de la materia orgánica particulada, la capa de 10 a $20 \mathrm{~cm}$ es donde se presentaría la mayor cantidad de materia orgánica estable o humificada, mientras que la capa intermedia sería una capa de transición (Elliott y Cambardella, 1991).

En cada lote seleccionado se realizaron tres muestreos para obtener tres muestras no disturbadas por cada profundidad y una muestra disturbada a cada profundidad. En cada punto de muestreo se recolectaron cinco submuestras de suelo por profundidad para conformar la muestra compuesta del punto. Dicho submuestreo se realizó en cruz sobre el punto de muestreo, donde la distancia de los tramos de la cruz es $1 \mathrm{~m}$. Las muestras no disturbadas se extrajeron con un muestreador Eijkelkamp (Eijkelkamp Soil \& Water, Giesbeek, Holanda) de cilindros metálicos de $98,175 \mathrm{~cm}^{3}$, siendo tomados tres a cada profundidad en cada punto de muestreo.

La caracterización de cada suelo evaluado se realizó con el análisis de algunas propiedades físicas y químicas, que permitió efectuar una adecuada interpretación de los resultados obtenidos en el análisis del CO. Las metodologías empleadas se presentan en la tabla 1.

El análisis del contenido de carbono orgánico total (COT) se realizó en muestras compuestas disturbadas para cada profundidad; secadas al aire y debidamente tamizadas, mediante el método de digestión y oxidación ácida en húmedo de Walkley y Black con medición colorimétrica mediante spectrofotometría (IGAC, 2006b). 
Tabla 1. Metodologías utilizadas para evaluar las propiedades físico-químicas del suelo.

\begin{tabular}{|l|l|}
\hline \multicolumn{1}{|c|}{ Propiedad } & \multicolumn{1}{c|}{ Método de análisis } \\
\hline $\begin{array}{l}\text { Distribución de partículas } \\
\text { minerales }\end{array}$ & Hidrómetro de Bouyoucos \\
\hline Densidad aparente & $\begin{array}{l}\text { Cilindros metálicos tipo Uhland modifi- } \\
\text { cados (muestreador Eijkelkamp) }\end{array}$ \\
\hline pH & $\begin{array}{l}\text { Potenciómetro en suspensión de } \\
\text { suelo con agua en relación 1:1 para } \\
\text { todas las muestras. }\end{array}$ \\
\hline $\begin{array}{l}\text { Capacidad de intercambio } \\
\text { catiónico }\end{array}$ & Acetato de amonio 1 N a pH 7 \\
\hline Conductividad eléctrica & $\begin{array}{l}\text { Conductímetro en suspensión de } \\
\text { suelo con agua en relación 1:1 para } \\
\text { todas las muestras }\end{array}$ \\
\hline $\begin{array}{l}\text { Saturación de bases } \\
\text { intercambiables }\end{array}$ & Acetato de amonio 1 N a pH 7 \\
\hline
\end{tabular}

La metodología utilizada para el fraccionamiento físico de la MOS, fue el método de suspensión y agitación en agua con tamizado, empleando tamices de 2,36 mm y 0,053 mm. Posterior a la separación con tamices de la fracción ligera y la fracción pesada de la materia orgánica del suelo, se determinó el contenido de $\mathrm{CO}$ en cada una, mediante combustión seca en mufla a $580^{\circ} \mathrm{C}$ por $12 \mathrm{~h}$ (IGAC, 2006b).

El carbono de la biomasa microbiana del suelo (CO de $\mathrm{BM}$ ), se midió en forma indirecta utilizando el método de respiración inducida por sustrato (glucosa), en muestras de suelo disturbadas que se refrigeraron a $4^{\circ} \mathrm{C}$ desde el muestreo hasta el momento del análisis (Lozano, 2005). Se calculó el índice de humificación como el cociente del carbono orgánico de la fracción pesada (CO de $\mathrm{FH}$ ), el carbono orgánico total (COT) y se expresó en porcentaje (IGAC, 2006b).

Se realizaron pruebas de normalidad y homogeneidad con un análisis de ANOVA, aquellas que no cumplieron los supuestos se efectuó un análisis no paramétrico de Kruskal-Wallis. Además, se realizó una prueba de contraste de diferencia mínima significativa con un nivel de confianza del $95 \%$ o por interpretación de la gráfica de bigotes. Para explicar la relación de variables se empleó análisis multivariado de componentes principales (ACP). Se utilizó el Excel (Microsoft ${ }^{\circledR}$, Washington, DC) para organizar las matrices de datos y los análisis se hicieron con Statgrafics v. 5.1 (Statgrafics Net, Madrid, España).

\section{RESULTADOS Y DISCUSIÓN}

Las propiedades químicas como $\mathrm{pH}$, conductividad eléctrica (CE), capacidad de intercambio catiónico (CIC) y saturación de bases intercambiables (SB), son las ideales para interpretar las condiciones del suelo como medio químico donde se desarrollan un conjunto de procesos físico-químicos, tales como oxidación de materia orgánica (Lozano et al., 2005). Se encontró en el suelo de clima frío un rango comprendido entre 5,07 a 6,99 de $\mathrm{pH}$ (Fig. 1) en todas las profundidades y usos del suelo. Es destacable que en el uso de suelo bosque y pastura, la acidez va de moderada a fuerte, mientras que en el uso de cultivo intensivo es neutro, lo cual evidencia la práctica del encalamiento. Se analizó que el suelo tiene muy buena capacidad de intercambio catiónico (CIC) con predominio de bases intercambiables y no hay problemas de salinidad (Fig. 1).

Las propiedades químicas del suelo de clima cálido (Fig. 2), muestran que son de reacción más ácido en comparación de los suelos de clima frío, con valores de 4,81 a 5,71, es decir, que las condiciones van de muy fuertemente ácido a moderadamente ácido. En el suelo de clima cálido hay mayor variabilidad en sus distintas propiedades químicas, destacando que la capacidad de intercambio catiónico y la saturación de bases intercambiables varían de alta hasta baja.

Las condiciones físico-químicas del suelo afectan el $\mathrm{COS}$, siendo el $\mathrm{pH}$ del suelo muy influyente sobre la cantidad y calidad del mismo. A su vez, la disponibilidad de nutrientes para los microorganismos descomponedores, especialmente las bases intercambiables $\mathrm{Ca}, \mathrm{Mg}$ y $\mathrm{K}$, también tienen una importancia señalada por muchos estudios sobre la dinámica del COS (Alexander, 1998; Varela y Rueda, 2010).

$\mathrm{Al}$ interpretar los resultados del contenido de arenas, arcillas y limos (Tabs. 2 y 3), se observa que el suelo del clima frío presenta texturas medias a moderadamente gruesas, ya que las arenas se presentan en un rango de 476,9 a $676,9 \mathrm{~g} \mathrm{~kg}^{-1}$, las arcillas de 153,8 a $253,8 \mathrm{~g} \mathrm{~kg}^{-1}$ y los limos de 116,0 a 269,3 $\mathrm{g} \mathrm{kg}^{-1}$ lo cual representa un predominio de las arenas. En el suelo de clima cálido, se presentan texturas finas a medias, ya que las arenas se presentan en un rango de 19,5 a $459,5 \mathrm{~g} \mathrm{~kg}^{-1}$, las arcillas de 253,8 a 597,8 $\mathrm{g} \mathrm{kg}^{-1}$ y los limos de 249,3 a $453,3 \mathrm{~g} \mathrm{~kg}^{-1}$ lo cual representa una presencia importante de arcillas. 

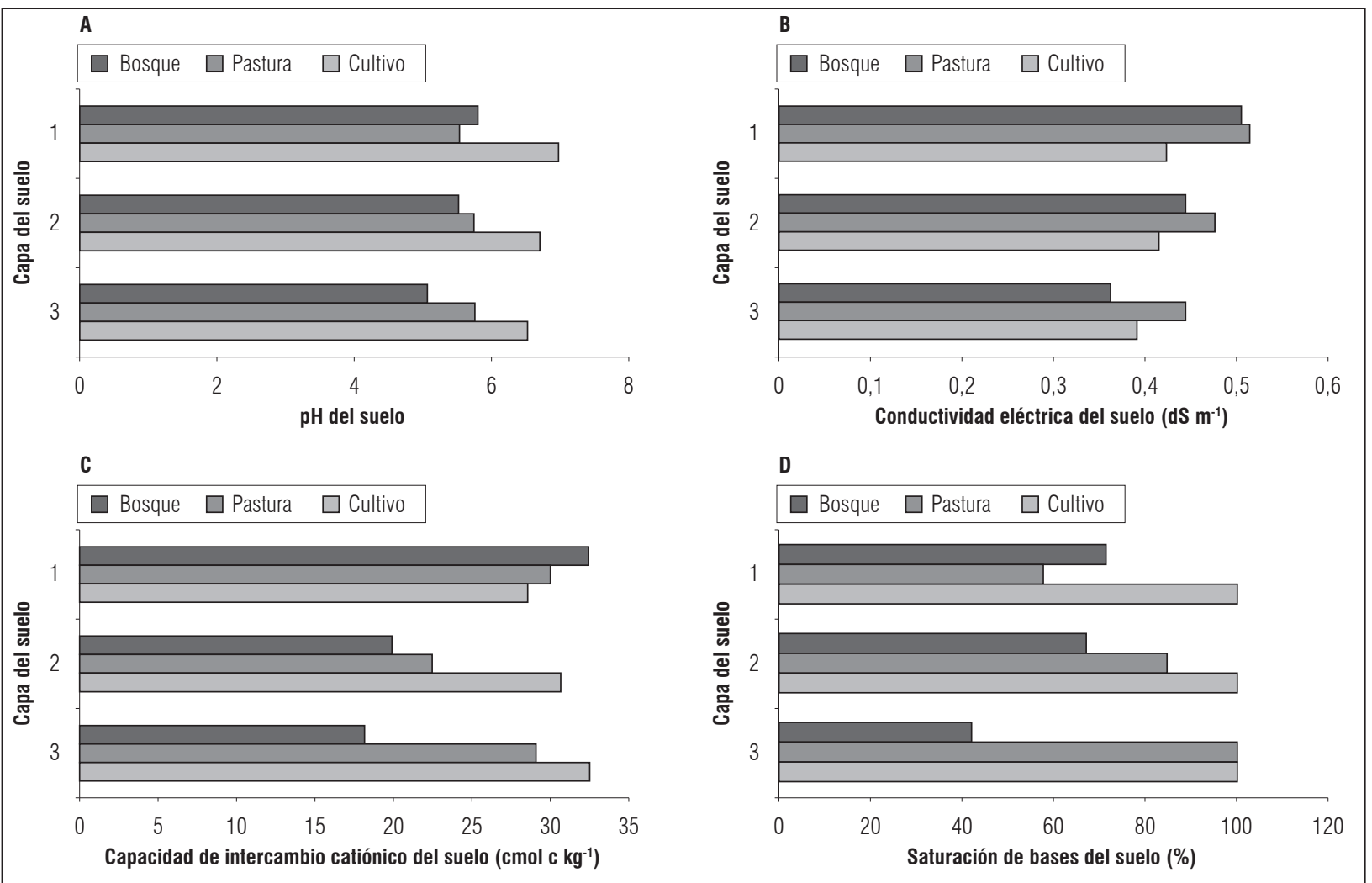

Figura 1. Propiedades químicas de suelos de clima frío en Pamplona, Colombia a diferentes profundidades y usos. Capa 1: 0 a $5 \mathrm{~cm}$; capa 2: 5 a $10 \mathrm{~cm}$ y capa $3: 10$ a $20 \mathrm{~cm}$.

Tabla 2. Distribución de tamaño de partículas minerales $\left(\mathrm{g} \mathrm{kg}^{-1}\right)$, clase textural y densidad aparente-Da $\left(\mathrm{Mg} \mathrm{m} \mathrm{m}^{-3}\right)$ en suelos de clima frío en Pamplona, Colombia, a diferentes profundidades y usos.

\begin{tabular}{|c|c|c|c|c|c|c|}
\hline Uso del suelo & Capa & Arenas & Arcillas & Limos & Clase textural & $\mathrm{Da}$ \\
\hline \multirow{3}{*}{ Bosque } & 1 & $\begin{array}{l}616,9 \\
(11,5) \\
\end{array}$ & $\begin{array}{l}153,8 \\
(23,1) \\
\end{array}$ & $\begin{array}{l}229,3 \\
(11,5) \\
\end{array}$ & Franco arenoso & $\begin{array}{c}1,03 \\
(0,05) \\
\end{array}$ \\
\hline & 2 & $\begin{array}{l}636,9 \\
(23,1) \\
\end{array}$ & $\begin{array}{l}153,8 \\
(23,1) \\
\end{array}$ & $\begin{array}{l}209,3 \\
(46,2) \\
\end{array}$ & Franco arenoso & $\begin{array}{c}1,08 \\
(0,04) \\
\end{array}$ \\
\hline & 3 & $\begin{array}{l}476,9 \\
(11,5) \\
\end{array}$ & $\begin{array}{l}253,8 \\
(41,6) \\
\end{array}$ & $\begin{array}{l}269,3 \\
(30,6) \\
\end{array}$ & Franco & $\begin{array}{c}1,03 \\
(0,05) \\
\end{array}$ \\
\hline \multirow{3}{*}{ Pastura } & 1 & $\begin{array}{l}670,2 \\
(30,6)\end{array}$ & $\begin{array}{l}153,8 \\
(11,5)\end{array}$ & $\begin{array}{l}176,0 \\
(40,0)\end{array}$ & Franco arenoso & $\begin{array}{c}1,34 \\
(0,02)\end{array}$ \\
\hline & 2 & $\begin{array}{l}650,2 \\
(30,6)\end{array}$ & $\begin{array}{l}207,1 \\
(11,5)\end{array}$ & $\begin{array}{l}142,7 \\
(41,6)\end{array}$ & $\begin{array}{c}\text { Franco arcillo } \\
\text { limoso }\end{array}$ & $\begin{array}{c}1,54 \\
(0,06)\end{array}$ \\
\hline & 3 & $\begin{array}{l}676,9 \\
(30,6)\end{array}$ & $\begin{array}{l}207,1 \\
(11,5)\end{array}$ & $\begin{array}{l}116,0 \\
(20,0) \\
\end{array}$ & $\begin{array}{c}\text { Franco arcillo } \\
\text { limoso }\end{array}$ & $\begin{array}{c}1,59 \\
(0,03) \\
\end{array}$ \\
\hline \multirow{3}{*}{ Cultivo intensivo } & 1 & $\begin{array}{l}530,2 \\
(11,5) \\
\end{array}$ & $\begin{array}{l}247,1 \\
(11,5) \\
\end{array}$ & $\begin{array}{l}222,7 \\
(11,5)\end{array}$ & Franco & $\begin{array}{c}1,40 \\
(0,04) \\
\end{array}$ \\
\hline & 2 & $\begin{array}{c}516,9 \\
(1,5)\end{array}$ & $\begin{array}{l}220,5 \\
(20,0) \\
\end{array}$ & $\begin{array}{l}262,7 \\
(23,1)\end{array}$ & Franco & $\begin{array}{c}1,53 \\
(0,05) \\
\end{array}$ \\
\hline & 3 & $\begin{array}{l}496,9 \\
(23,1)\end{array}$ & $\begin{array}{l}240,5 \\
(20,0)\end{array}$ & $\begin{array}{l}262,7 \\
(11,5)\end{array}$ & Franco & $\begin{array}{c}1,43 \\
(0,03)\end{array}$ \\
\hline
\end{tabular}

Media (desviación estándar) de $n=3$. 
Tabla 3. Distribución de tamaño de partículas minerales $\left(\mathrm{g} \mathrm{kg}^{-1}\right)$, clase textural y densidad aparente-Da (Mg m-3) en suelos de clima cálido en El Zulia, Colombia, a diferentes profundidades y usos.

\begin{tabular}{|c|c|c|c|c|c|c|}
\hline Uso del suelo & Capa & Arenas & Arcillas & Limos & Clase textural & $\mathrm{Da}$ \\
\hline \multirow{3}{*}{ Bosque } & 1 & $\begin{array}{c}70,2 \\
(6,00)\end{array}$ & $\begin{array}{l}587,1 \\
(11,5)\end{array}$ & $\begin{array}{l}342,7 \\
(30,6)\end{array}$ & Arcilloso & $\begin{array}{c}1,14 \\
(0,03)\end{array}$ \\
\hline & 2 & $\begin{array}{l}19,5 \\
(0,0) \\
\end{array}$ & $\begin{array}{l}597,8 \\
(23,1) \\
\end{array}$ & $\begin{array}{l}382,7 \\
(23,1) \\
\end{array}$ & Arcilloso & $\begin{array}{r}1,39 \\
(0,03) \\
\end{array}$ \\
\hline & 3 & $\begin{array}{c}56,9 \\
(11,5)\end{array}$ & $\begin{array}{l}580,5 \\
(20,0)\end{array}$ & $\begin{array}{l}362,7 \\
(11,5)\end{array}$ & Arcilloso & $\begin{array}{c}1,62 \\
(0,02)\end{array}$ \\
\hline \multirow{3}{*}{$\begin{array}{l}\text { Palma de aceite } \\
\text { y pastura }\end{array}$} & 1 & $\begin{array}{l}386,2 \\
(11,5)\end{array}$ & $\begin{array}{l}299,1 \\
(30,6)\end{array}$ & $\begin{array}{l}314,7 \\
(41,6)\end{array}$ & Franco arcilloso & $\begin{array}{c}1,17 \\
(0,03)\end{array}$ \\
\hline & 2 & $\begin{array}{l}430,2 \\
(23,1) \\
\end{array}$ & $\begin{array}{l}253,8 \\
(11,5) \\
\end{array}$ & $\begin{array}{l}316,0 \\
(34,6) \\
\end{array}$ & Franco & $\begin{array}{c}1,51 \\
(0,02) \\
\end{array}$ \\
\hline & 3 & $\begin{array}{l}459,5 \\
(20,0) \\
\end{array}$ & $\begin{array}{l}291,1 \\
(23,1)\end{array}$ & $\begin{array}{l}249,3 \\
(11,5)\end{array}$ & Franco arcilloso & $\begin{array}{c}1,63 \\
(0,01) \\
\end{array}$ \\
\hline \multirow{3}{*}{ Cultivo intensivo } & 1 & $\begin{array}{l}410,2 \\
(30,6)\end{array}$ & $\begin{array}{l}253,8 \\
(11,5)\end{array}$ & $\begin{array}{l}336,0 \\
(20,0)\end{array}$ & Franco & $\begin{array}{r}1,43 \\
(0,04) \\
\end{array}$ \\
\hline & 2 & $\begin{array}{l}383,5 \\
(52,9) \\
\end{array}$ & $\begin{array}{l}287,1 \\
(11,5) \\
\end{array}$ & $\begin{array}{l}329,3 \\
(50,3) \\
\end{array}$ & Franco & $\begin{array}{c}1,45 \\
(0,05) \\
\end{array}$ \\
\hline & 3 & $\begin{array}{l}292,9 \\
(11,5)\end{array}$ & $\begin{array}{l}253,8 \\
(11,5)\end{array}$ & $\begin{array}{l}453,3 \\
(11,5)\end{array}$ & Franco & $\begin{array}{r}1,59 \\
(0,03)\end{array}$ \\
\hline
\end{tabular}

Media (desviación estándar) de $n=3$.

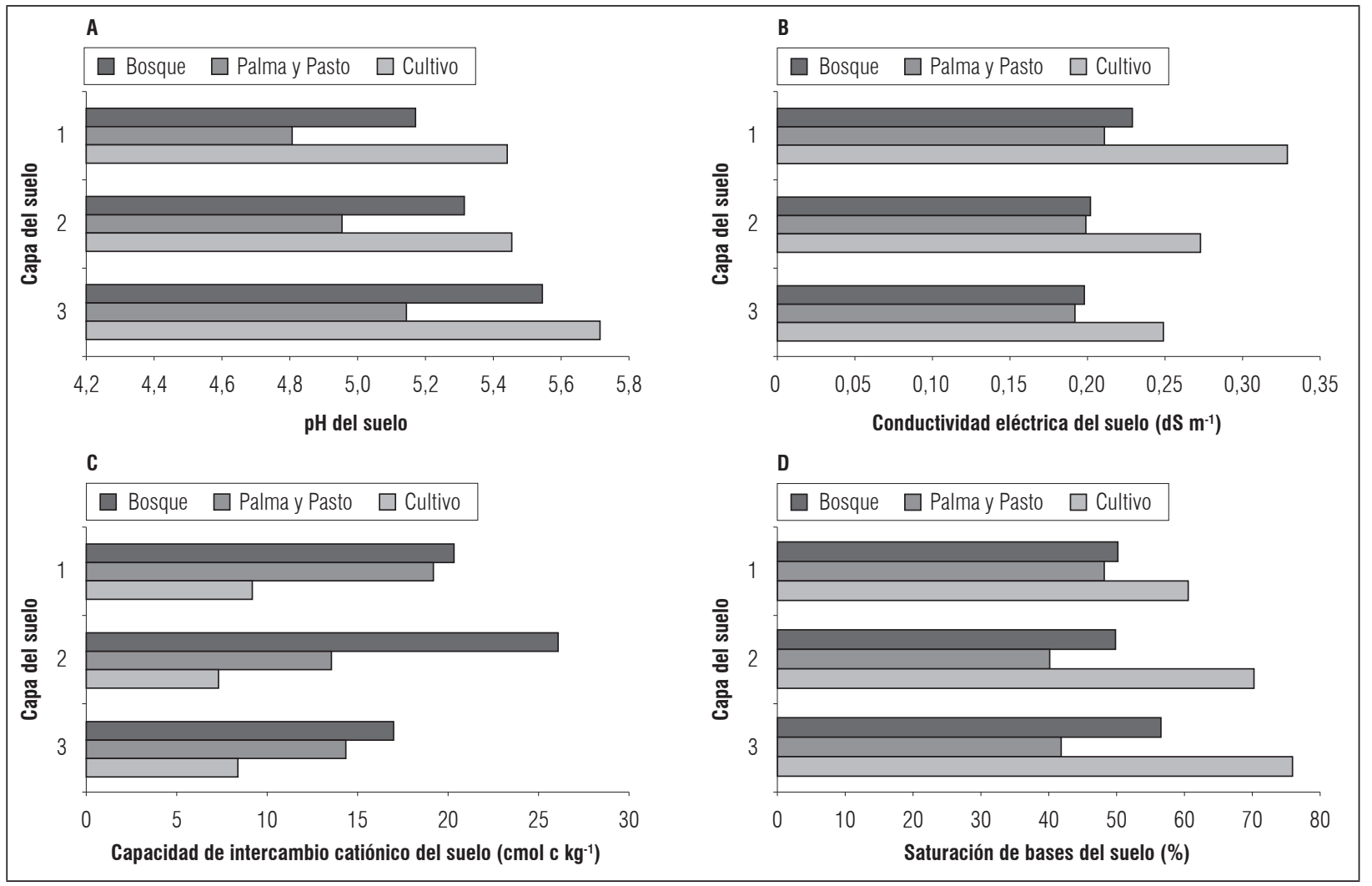

Figura 2. Propiedades químicas de suelos de clima cálido en El Zulia, Colombia a diferentes profundidades y usos. Capa 1: 0 a $5 \mathrm{~cm}$; capa 2: 5 a $10 \mathrm{~cm}$ y capa 3: 10 a $20 \mathrm{~cm}$. A. ph; B. Conductividad; C. Capacidad de intercambio catiónico y D. Saturación de bases del suelo. 
La densidad aparente (Da) se interpreta considerando el tipo de textura, encontrándose que, en suelos con texturas gruesas o moderadamente gruesas, valores inferiores a 1,60 son favorable y para texturas medias valores inferiores a 1,40 son favorables (Pla, 2010). En este sentido, se observó que el lote en uso de bosque en clima frío tiene Da de 1,03 a 1,08 y 1,03 en la primera y tercera capa de profundidad respectivamente, lo cual representa buenas condiciones físicas. En el caso de los suelos pastura y cultivo, la $\mathrm{Da}$ de la primera capa presenta valor favorable, 1,34 y 1,40, respectivamente. Pero en la segunda y tercera capa de ambos suelos, la Da es mayor a 1,40 lo cual expresa limitación física debido a reducción del espacio poroso del suelo.

En clima cálido, los suelos bosque y palma de aceite con pastura presentaron valores de Da favorables en la capa superficial, 1,14 y 1,17, respectivamente. Pero estos suelos presentaron signos de compactación a partir de $5 \mathrm{~cm}$ de profundidad (por el esfuerzo en la toma de muestras). En el caso del suelo cultivo intensivo se encontró alta compactación en las tres capas de profundidad, significando serios problemas por perdida de espacio poroso de aireación y dificultad para el desarrollo de raíces y microorganismos, lo cual afecta el contenido de COT.

Los resultados del contenido de COT en términos de masa ( $\mathrm{mg} \mathrm{ha}^{-1}$ ) no cumplieron los supuestos de normalidad y homogeneidad de varianza y el análisis no paramétrico de Kruskal-Wallis presentó un efecto estadísticamente significativo $(P=0,000)$ ejercido por los factores clima y uso del suelo.
El contenido de COT y uso de los suelos en la zona de clima cálido fueron diferenciados estadísticamente en dos grupos (Fig. 3). El primero de mayor contenido de COT está integrado por la implementación palma-pasto. El segundo de menor contenido de COT y lo integra los suelos de cultivo y bosque.

Estos resultados concordantes con los resultados de numerosos autores, que han encontrado contenidos superiores de COT en suelos con pasturas, con respecto a usos de cultivos intensivos de ciclo corto. Sánchez (1976) reportó hace 41 años la disminución del contenido de COT debido a la desforestación y uso agrícola del suelo en cultivos intensivos, ya que se genera un desbalance entre la adición de materia orgánica al suelo y la velocidad de descomposición.

Los valores medidos en laboratorio del carbono orgánico de la biomasa microbiana del suelo (CO de BM), son una medida indirecta de uno de los compartimientos más importantes del COS, representado por la materia orgánica viva o biota microscópica (Arias et al., 2012). Para interpretar estos resultados se realizó el ANOVA una vez que se comprobó el cumplimiento de los supuestos estadísticos.

Se evidencia diferencia significativa $(P=0,000)$ para el comportamiento del $\mathrm{CO}$ de BM debido a los factores clima, profundidad y uso. Así también se observa que hay efecto significativo de todas las interacciones posibles entre los factores evaluados sobre la respuesta de la CO de BM del suelo (Fig. 4), coincidencia con estudios previos (Delgado y España, 2000), que han señalado a la biomasa microbiana del suelo como un
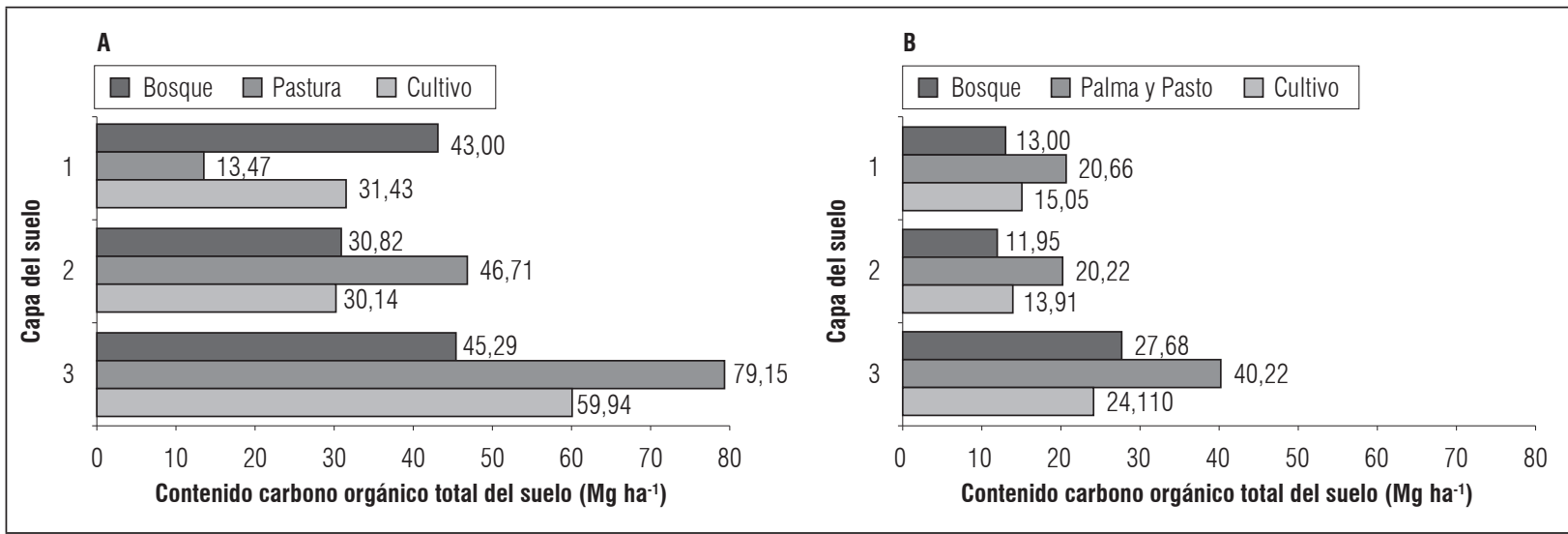

Figura 3. Contenido de carbono orgánico total en dos zonas de Norte de Santander (Colombia) según el tipo de uso y profundidad del suelo. A. Clima frío (Pamplona) y B. Clima cálido (El Zulia). Capa 1: 0 a $5 \mathrm{~cm}$; capa 2: 5 a $10 \mathrm{~cm}$ y capa 3: 10 a $20 \mathrm{~cm}$. 


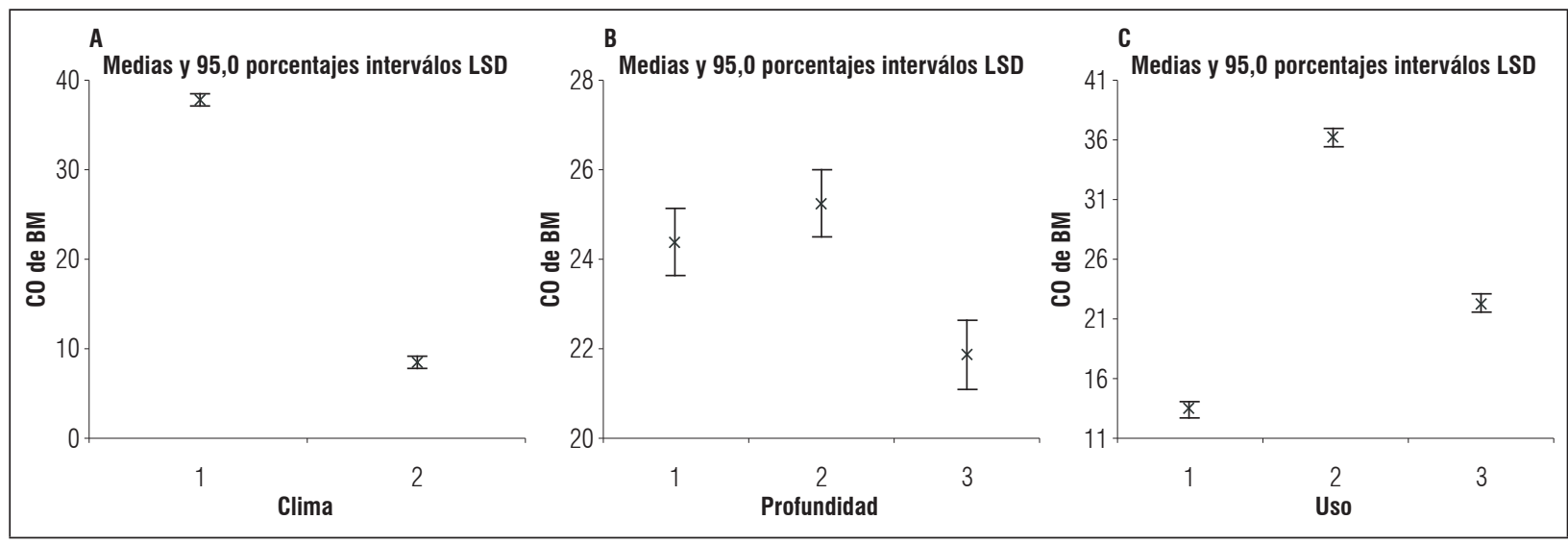

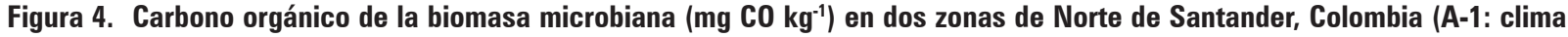
frío-Pamplona; 2: clima cálido-El Zulia), según la profundidad del suelo (B-1: 0 a $5 \mathrm{~cm} ; 2: 5$ a $10 \mathrm{~cm} ; 3: 10$ a $20 \mathrm{~cm}$ ) y el tipo de uso (C-1: bosque; 2: pastura y palma-pasto; 3: cultivo). Prueba de contraste de diferencia mínima significativa con un nivel de confianza del $95 \%$.

parámetro muy sensible para el estudio de la dinámica del COS, ya que al ser la descomposición de la MOS un proceso eminentemente microbiano, entonces medir la actividad microbiana del suelo a través de su respiración resulta ser un indicador confiable (Albaladejo et al., 2009).

Para encontrar los contrastes de respuestas estadísticamente significativas se procedió a realizar la prueba de la mínima diferencia significativa (DMS) (Fig. 4), y se constata que por efecto del clima es mayor el CO de BM en clima frío. Con respecto al efecto del factor profundidad del suelo, observados grupos homogéneos, el primero lo integran la capa de 0 a $5 \mathrm{~cm}$ conjuntamente con la capa de 5 a $10 \mathrm{~cm}$, y expresan los mayores contenidos de $\mathrm{CO}$ de $\mathrm{BM}$, estando el segundo grupo integrado por la capa de 10 a $20 \mathrm{~cm}$ donde se presentan los menores contenidos de CO de BM.

Para el efecto del uso del suelo sobre el CO de BM (Fig. 4), se encuentran tres grupos significativamente diferentes, el mayor contenido para el uso de pasturas y palma - pasto, seguido de cultivos intensivos y el menor para suelos de bosque.

En los resultados de carbono orgánico de la fracción ligera ( $\mathrm{CO}$ de $\mathrm{FL}$ ) fue necesario un análisis no paramétrico de Kruskal-Wallis, encontrando diferencia estadística significativa para el factor clima. No se presentó diferencias para los demás factores, tipo de uso y profundidad.

Los resultados indican que el clima cálido contiene menor CO de la FL en el suelo y el clima frío favorece un mayor contenido de $\mathrm{CO}$ de la FL en el suelo (Fig. 5). Al respecto Lavelle (1997), señala que en climas cálidos y húmedos, se favorece la existencia de una fauna del suelo muy diversa y activa que produce la rápida descomposición de la MOS, de esta manera no hay oportunidad a la acumulación de cantidad importante de materia orgánica particulada y por lo tanto el CO de la fracción ligera será menor.

A pesar de no ser estadísticamente significativo el efecto de la profundidad del suelo en el contenido de $\mathrm{CO}$ de la FL (Fig. 5), se encuentran estudios sobre el COS en los compartimientos de la MOS en el trópico (Figueiredo et al., 2010; Oliveira et al., 2016), en los cuales han registrado una clara estratificación en la distribución del $\mathrm{CO}$ en la FL en el perfil del suelo. Siendo lo más dominante encontrar que el mayor contenido de CO de la FL está en la capa superficial de 0 a $5 \mathrm{~cm}$ de profundidad, en condiciones de uso de bosque y pasturas.

Para el carbono orgánico de la fracción húmica (CO de la FH en $\mathrm{mg} \mathrm{ha}^{-1}$ ) el análisis de ANOVA mostró un efecto significativo debido al clima y la profundidad del suelo. También se observa que hay efecto significativo en la interacción entre los factores clima y uso del suelo sobre la respuesta del $\mathrm{CO}$ de la $\mathrm{FH}$, tal y como lo señalan otros estudios (Six et al., 2002). La prueba de contraste de medias indica que el contenido de $\mathrm{CO}$ de la FH (mg ha ${ }^{-1}$ ) es mayor en el clima frío (Fig. 6). Mientras que debido al efecto de la profundidad del suelo, se observan dos grupos homogéneos y significativamente diferentes entre sí. El primero lo 


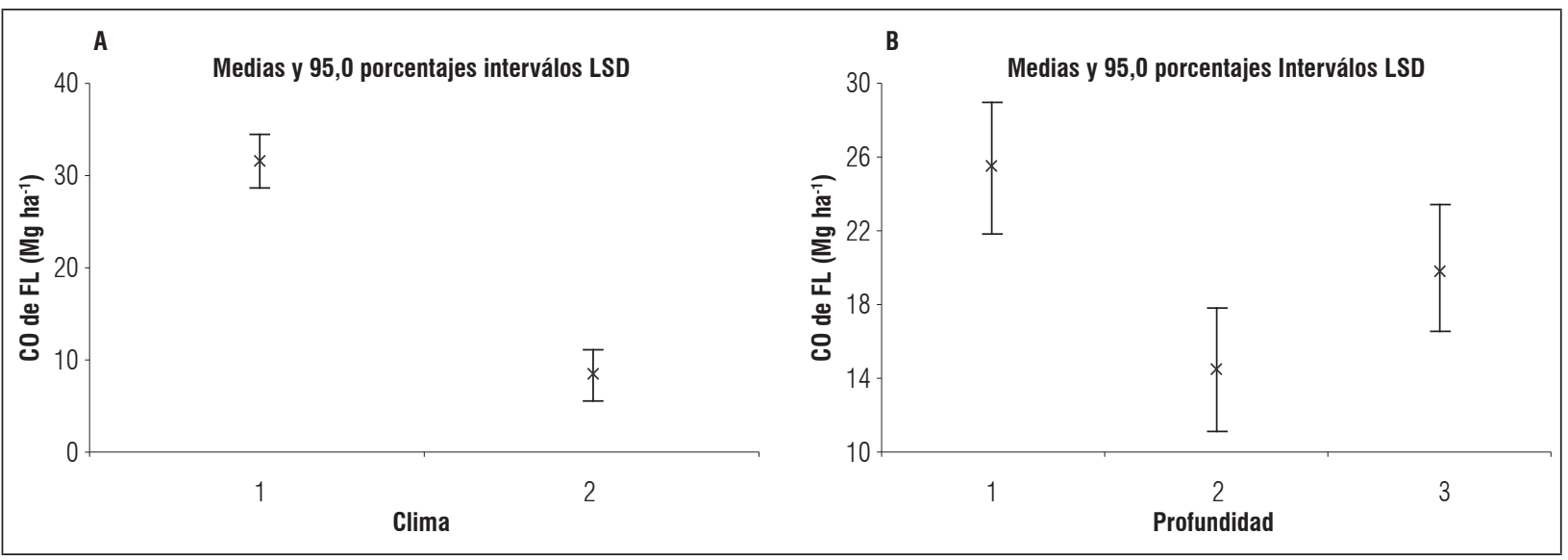

Figura 5. Carbono orgánico de la fracción ligera en dos zonas de Norte de Santander, Colombia (A-1: clima frío-Pamplona; 2: clima cálido-El Zulia) y profundidad del suelo (B-1: 0 a $5 \mathrm{~cm} ; 2: 5$ a $10 \mathrm{~cm} ; 3: 10$ a $20 \mathrm{~cm}$ ). Prueba de contraste de diferencia mínima significativa con un nivel de confianza del $95 \%$.

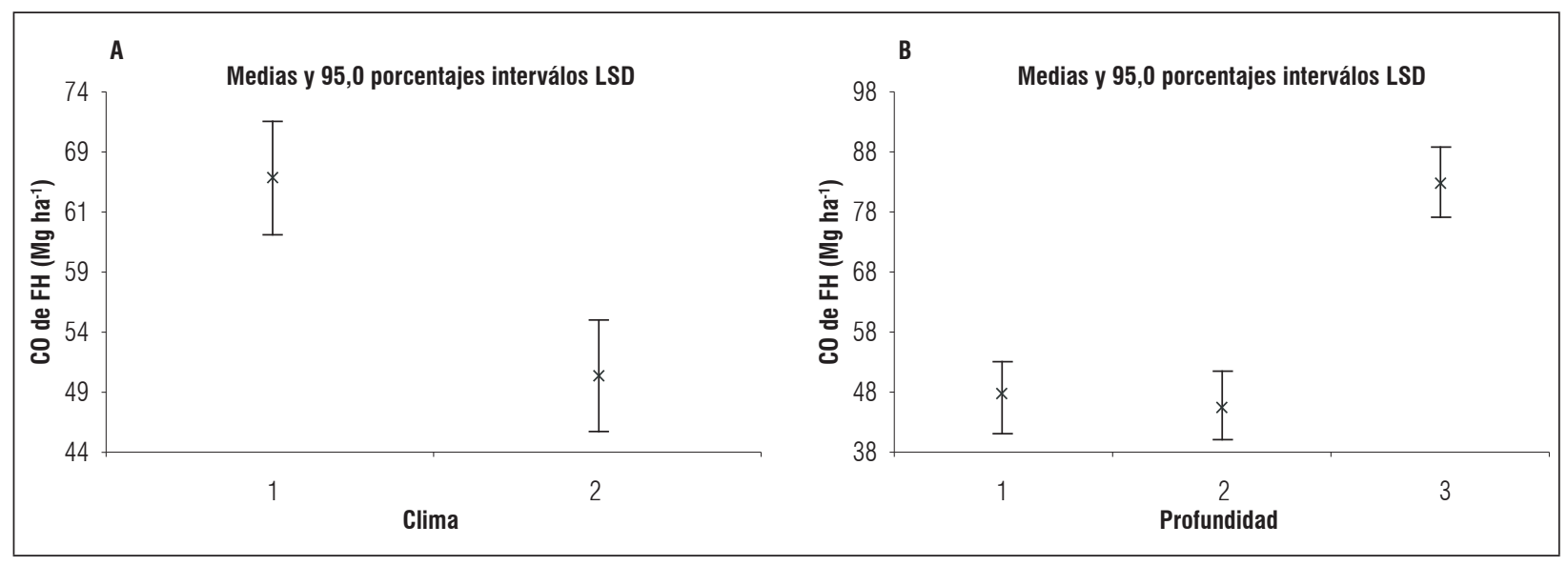

Figura 6. Carbono orgánico de la fracción húmica en dos zonas de Norte de Santander, Colombia (A-1: clima frío-Pamplona; 2: clima cálido-El Zulia) y profundidad del suelo (B-1: 0 a 5 cm; 2: 5 a $10 \mathrm{~cm} ; 3: 10$ a $20 \mathrm{~cm}$ ). Prueba de contraste de diferencia mínima significativa con un nivel de confianza del $95 \%$.

integra la capa de 10 a $20 \mathrm{~cm}$ de profundidad con el mayor contenido de CO de la FH en masa. El segundo grupo con menor contenido de $\mathrm{CO}$ de la FH en masa lo integran la capa de 5 a $10 \mathrm{~cm}$ y la capa de 0 a $5 \mathrm{~cm}$. Debido al efecto significativo de la interacción clima y uso, se debe decir que el mayor contenido de $\mathrm{CO}$ de la FH expresado en masa se presenta en clima frío y en la profundidad del suelo 10 a $20 \mathrm{~cm}$.

Con respecto al índice de humificación fue significativamente mayor en el clima cálido (Fig. 7). Esto representa un resultado altamente relevante, puesto que indica que el suelo en clima cálido, indistintamente del uso y la profundidad, tiene mayor capacidad de descomponer, mineralizar y humificar la MOS. Lo cual es consistente, con los fundamentos expresados, en temperaturas cálidas con adecuadas condiciones de humedad, los microorganismos del suelo son más activos en su trabajo de descomposición de la MOS, ayudados por una fauna del suelo diversa y dinámica. También influye que el suelo en el clima cálido, presentó los mayores contenidos de partículas minerales finas, lo cual favorece la formación de complejos arcillo-húmicos que aumentan la estabilización química y el periodo de residencia en el suelo del carbono orgánico (Six et al., 2002). 


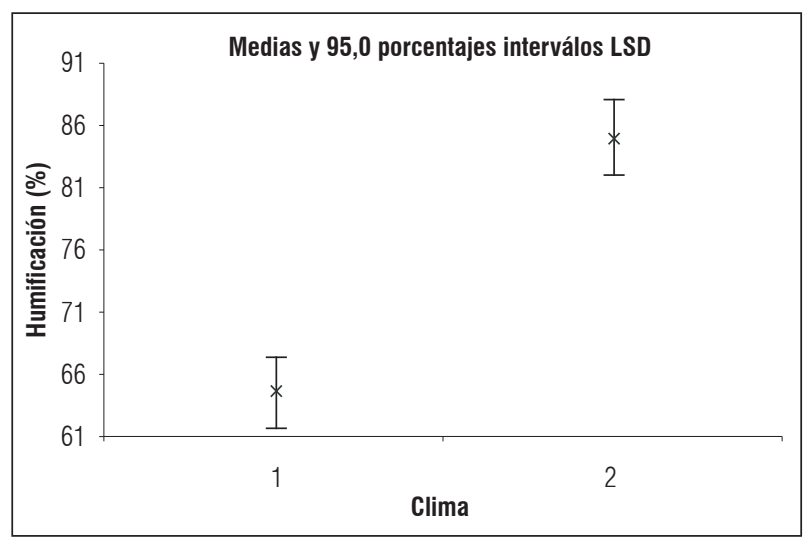

Figura 7. Índice de humificación en dos zonas de Norte de Santander, Colombia (1. Clima frío, Pamplona; 2. Clima cálido, El Zulia). Prueba de contraste de diferencia mínima significativa con un nivel de confianza del $95 \%$.

El análisis multivariado de componentes principales mostró que son tres las combinaciones de variables capaces de explicar la mayoría de la variabilidad de los datos, estas conforman los tres componentes con autovalor más altos y que conjuntamente explican el $75,55 \%$ de la variabilidad en los datos originales (Tab. 4). El otro $24,45 \%$ de la variabilidad lo expresan el resto de 13 combinaciones de variables que no se consideran importantes y por ello no se toman en cuenta.

En este sentido, se interpreta que los tres componentes principales encontrados revelan tres procesos muy importantes de la dinámica del COS y su respuesta a los factores clima, uso de la tierra y profundidad del suelo. El primer componente reúne las variables físicas Arcillas, Limos y Da, como parámetros que sirven para explicar la relación de las condiciones físicas del suelo con los procesos de estabilización del COS. El segundo componente reúne las variables químicas pH, SB, CIC y CE, como propiedades químicas capaces de explicar la relación de la descomposición de la MOS con las condiciones químicas del medio edáfico. El tercer componente agrupa las variables COT, CO de la FL y $\mathrm{CO}$ de la FH, lo cual explica la interrelación existente entre los distintos compartimientos del COS y sirve para reforzar la justificación de medir el $\mathrm{CO}$ en cada compartimiento del suelo para verdaderamente entender la dinámica del COS.

Al realizar el análisis se obtuvo que las variables arenas, CO de BM e Índice de humificación participan en las combinaciones de variables que tienen baja capacidad en explicar la mayoría de la variabilidad de los datos de este estudio.
Tabla 4. Análisis de componentes principales del análisis multivariado.

\begin{tabular}{|c|c|c|c|}
\hline $\begin{array}{c}\text { Número del } \\
\text { componente }\end{array}$ & Autovalor & $\begin{array}{c}\text { Porcentaje de } \\
\text { varianza }\end{array}$ & $\begin{array}{c}\text { Porcentaje } \\
\text { acumulado }\end{array}$ \\
\hline 1 & 7,49397 & 46,837 & 46,837 \\
\hline 2 & 2,84214 & 17,763 & 64,601 \\
\hline 3 & 1,75204 & 10,950 & 75,551 \\
\hline
\end{tabular}

Componente 1: arcillas, limos y densidad aparente; 2: pH, saturación de bases, capacidad de intercambio catiónico y conductividad eléctrica; 3: carbono orgánico total; carbono orgánico de la fracción ligera y carbono orgánico de la fracción húmica.

\section{CONCLUSIONES}

Los resultados permiten decir que en general los suelos de clima frío son capaces de almacenar mayor contenido de carbono en todos los compartimientos (COT, CO de BM, CO de FL y CO de FH). Siendo el uso del suelo bosque y pastura, la condición más favorable para el almacenamiento de COS, permitiendo un comportamiento como sumidero de carbono. Mientras que el uso del suelo con cultivo intensivo genera un menor contenido de COT, CO de FL y CO de BM, lo cual demuestra un comportamiento como emisor de carbono a la atmosfera.

El índice de humificación es significativamente mayor en el clima cálido. Esto representa un resultado altamente relevante, puesto que indica que el suelo en clima cálido, indistintamente del uso y la profundidad, tiene mayor capacidad de descomponer, mineralizar y humificar la MOS, lo cual representa una importante capacidad de ser sumidero de COS, si los aportes de MOS son suficientemente altos y continuos.

En este estudio se encontró que las variables físicas (arenas, limos y $\mathrm{Da}$ ) conjuntamente con las variables químicas ( $\mathrm{pH}, \mathrm{CE}, \mathrm{CIC}$ y SB), son útiles para estudiar el comportamiento del COS y su respuesta ante los factores clima, tipo de uso y profundidad del suelo.

Para estudiar la dinámica del COS es necesario medir su contenido en los distintos compartimientos de la materia orgánica del suelo, como son: la fracción ligera, la fracción húmica y la biomasa microbiana.

Conflicto de intereses: el manuscrito fue preparado y revisado con la participación de los autores, quienes declaran no tener algún conflicto de interés que coloquen en riesgo la validez de los resultados aquí presentados. 


\section{REFERENCIAS BIBLIOGRÁFICAS}

Albaladejo, J., M. Martínez, M. Almagro, A. Ruiz y R. Ortiz. 2009. Factores de control en la dinámica del carbono orgánico de los suelos de la región de Murcia. pp. 155-158. En: Congreso Internacional sobre Desertificación en memoria del profesor John B. Thornes. Universidad de Murcia, Murcia, España.

Alexander, M. 1998. Principles and applications of soil microbiology. 6a ed. Pearson Prentice Hall, Upper Saddle River, NJ, USA.

Arias, H., E. Malavassi, B. Alvarado y R. Chazdon. 2012. Biomasa sobre el suelo y carbono orgánico en el suelo en cuatro estadios de sucesión de bosques de la Península de Osa, Costa Rica. Rev. For. Mesoam. Kurú 9(22), 22-31. Doi: 10.18845/rfmk.v15i36.3422

Delgado, R. y M. España. 2000. Evaluación de la biomasa microbiana por los métodos de fumigación-incubación y fumigación-extracción y su relación con la disponibilidad de nitrógeno en suelos de Venezuela. Agron. Trop. 50(4), 537-551.

Elliott, E. y C. Cambardella. 1991. Physical separation of soil organic matter. Agric Ecosyst. Environ. 34, 407419. Doi: 10.1016/0167-8809(91)90124-G

Ferreira, E., M. Bustamante, D. Resck, C. Figueiredo, A. Pinto y J. Malaquias. 2016. Carbon stocks in compartments of soil organic matter 31 years after substitution of Native Cerrado vegetation by agroecosystems. Rev. Bras. Ciênc. Solo 40, e0150059. Doi: 10.1590/18069657rbcs20150059

Figueiredo, C., D. Resck y M. Carneiro. 2010. Labile and stable fractions of soil organic matter under management systems and Native Cerrado. Rev. Bras. Ciênc. Solo 34, 907-916. Doi: 10.1590/S0100-06832010000300032

Ghimire, R., S. Lamichhane, B. Acharya, P. Bista y U. Sainju. 2017. Tillage, crop residue, and nutrient management effects on soil organic carbon in rice-based cropping systems: a review. J. Integr. Agric. 16, 1-15. Doi: 10.1016/S2095-3119(16)61337-0

Gobernación de Norte de Santander. 2016. Plan de Desarrollo del departamento Norte de Santander presentado para el periodo 2016-2019. Cúcuta, Colombia.

IGAC. 2006a. Estudio general de suelos y zonificación de tierras del departamento Norte de Santander. Instituto Geográfico Agustín Codazzi, Bogotá, Colombia.

IGAC. 2006b. Métodos analíticos de laboratorio de suelos. $6^{a}$ ed. Instituto Geográfico Agustín Codazzi, Bogotá, Colombia.
Kassa, H., S. Dondeyne, J. Poesen, A. Frankl y J. Nyssen. 2017. Impact of deforestation on soil fertility, soil carbon and nitrogen stocks: the case of the Gacheb catchment in the White Nile Basin, Ethiopia. Agr. Ecosyst. Environ. 247, 273-282. Doi: 10.1016/j. agee.2017.06.034

Lavelle, P. 1997. Faunal activities and soil processes: adaptative strategies that determine ecosystem function. Adv. Ecol. Res. 27, 93-132. Doi: 10.1016/ S0065-2504(08)60007-0

Lozano, Z., R. Hernández y A. Ojeda. 2005. Manual de métodos para la evaluación de la calidad física, química y biológica de los suelos. Universidad Central de Venezuela, Caracas, Venezuela.

Macías, F. y M. Camps-Arbestain. 2010. Soil carbon sequestration in a changing environment. Mitig. Adapt. Strateg. Glob. Change 15, 511-529. Doi: 10.1007/ s11027-010-9231-4

Manlay, R., C. Feller y M. Swift. 2007. Historical evolution of soil organic matter concepts and their relationships with the fertility and sustainability of cropping systems. Agr. Ecosyst. Environ. 119, 217-233. Doi: 10.1016/j.agee.2006.07.011

Oliveira, F., J. Oliveira y F. Xavier. 2016. Changes in soil organic carbon fractions in response to cover crops in an orange orchard. Rev. Bras. Ciênc. Solo 40. Doi: 10.1590/18069657rbcs20150105

Olson, K., S. Ebelhar y J. Lang. 2014 Long-term effects of cover crops on crop yields, soil organic carbon stocks and sequestration. Open J. Soil Sci. 4, 284-292. Doi: 10.4236/ojss.2014.48030

Pla, I. 2010. Medición y evaluación de propiedades físicas de los suelos: dificultades y errores más frecuentes. I - Propiedades mecánicas. Suelos Ecuat. 40(2), 75-93.

Sánchez, P. 1976. Properties and management of the soils of the tropics. John Wiley and Sons, New York, NY, USA.

Six, J., R. Conant, E. Paul y K. Paustian. 2002. Stabilization mechanisms of soil organic matter: implications for c-saturation of soils. Plant Soil 241, 155-176. Doi: 10.1023/A:1016125726789

Ussiri, D. y R. Lal. 2013. Land management effects on carbon sequestration and soil properties in reclaimed farmland of Eastern Ohio, USA. Open J. Soil Sci. 3, 46-57. Doi: 10.4236/ojss.2013.31006

Varela, A. y D. Rueda. 2010. Organismos del suelo. pp. $397-$ 438. En: Burbano O., H. y F. Silva M. (eds.). Ciencia del suelo principios básicos. Sociedad Colombiana de la Ciencia del Suelo, Bogotá, Colombia. 